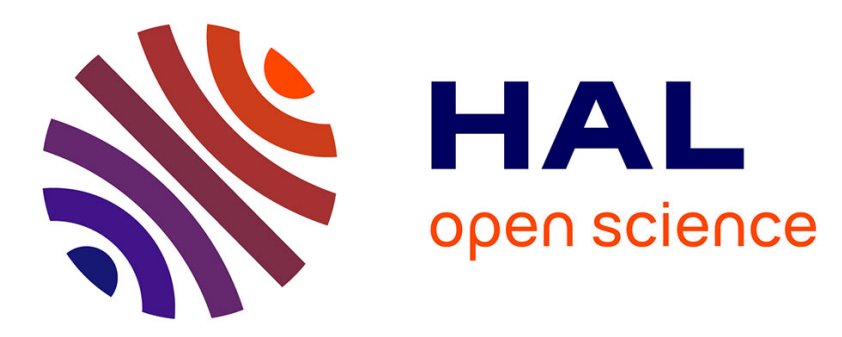

\title{
Vlasov modelling of parallel transport in a tokamak scrape-off layer
}

\author{
Giovanni Manfredi, Sever Adrian Hirstoaga, Stéphane Devaux
}

\section{To cite this version:}

Giovanni Manfredi, Sever Adrian Hirstoaga, Stéphane Devaux. Vlasov modelling of parallel transport in a tokamak scrape-off layer. Plasma Physics and Controlled Fusion, 2011, 53 (1), pp.015012. 10.1088/0741-3335/53/1/015012 . hal-00538153

\section{HAL Id: hal-00538153 \\ https://hal.science/hal-00538153}

Submitted on 22 Nov 2010

HAL is a multi-disciplinary open access archive for the deposit and dissemination of scientific research documents, whether they are published or not. The documents may come from teaching and research institutions in France or abroad, or from public or private research centers.
L'archive ouverte pluridisciplinaire HAL, est destinée au dépôt et à la diffusion de documents scientifiques de niveau recherche, publiés ou non, émanant des établissements d'enseignement et de recherche français ou étrangers, des laboratoires publics ou privés. 


\title{
Vlasov modelling of parallel transport in a tokamak scrape-off layer
}

\author{
G. Manfredi
}

Institut de Physique et Chimie des Matériaux, CNRS and Université de Strasbourg, BP 43, F-67034 Strasbourg, France

E-mail: Giovanni.Manfredi@ipcms.u-strasbg.fr

\section{S. Hirstoaga}

INRIA Nancy Grand-Est and Institut de Recherche en Mathématiques Avancées, 7 rue René Descartes, F-67084 Strasbourg, France

E-mail: hirstoaga@math.unistra.fr

\section{S. Devaux}

JET-EFDA, Culham Science Centre, OX14 3DB, Abingdon, United Kingdom

E-mail: Stephane.Devaux@ccfe.ac.uk

\begin{abstract}
A one-dimensional Vlasov-Poisson model is used to describe the parallel transport in a tokamak scrape-off layer. Thanks to a recently-developed 'asymptoticpreserving' numerical scheme, it is possible to lift numerical constraints on the time step and grid spacing, which are no longer limited by, respectively, the electron plasma period and Debye length. The Vlasov approach provides a good velocityspace resolution even in regions of low density. The model is applied to the study of parallel transport during edge-localized modes (ELMs), with particular emphasis on the particles and energy fluxes on the divertor plates. The numerical results are compared to analytical estimates based on a free-streaming model, with good general agreement. An interesting feature is the observation of an early electron energy flux, due to suprathermal electrons escaping the ions' attraction. In contrast, the long-time evolution is essentially quasi-neutral and dominated by the ion dynamics.
\end{abstract}




\section{Introduction}

One of the main challenges for future tokamak operation, such as ITER, is constituted by the large heat load on the divertor plates. The divertor surfaces are constantly bombarded with high-energy neutral and charged particles and may thus see their lifetime considerably reduced $[1,2]$. The erosion also releases high-Z impurities, which migrate towards the bulk plasma and, due to radiation, deteriorate its confinement [3]. In order to keep within reasonable limits the erosion of different wall materials, it is important to estimate the plasma characteristics in the scrape-off layer (SOL), i.e. the region outside the last closed magnetic surface (separatrix). Several numerical and analytical studies have addressed the problem of the transition between a hot plasma and a material surface, both for unmagnetized and magnetized plasmas $[4,5,6,7]$.

Most such studies focus on the static plasma-wall transition. However, transient events - such as edge-localized modes (ELMs) - routinely occur in the tokamak edge during H-mode confinement [8]. ELMs are plasma relaxations, presumably of MHD origin, which cause a sudden drop in density and temperature of the pedestal plasma, leading to a significant loss of the stored plasma energy $(5-20 \%)[9,10]$. Once the ELM-driven plasma pulse has crossed the magnetic separatrix, it travels mainly parallel to the magnetic field lines and ends up hitting the divertor plate, after a delay typically of the order of a few hundred microseconds. Such violent events can pose a serious threat to the long-time resistance of the divertor materials.

The SOL is a complex region where a multitude of physical and chemical processes take place, occurring on rather disparate space and time scale. A realistic description of the SOL thus requires sophisticated modelling that takes into account, among others, plasma turbulence, collisions and ionisation, impurity transport, and plasma-surface interactions. The corresponding simulations $[11,12]$ are computationally very costly, so that a thorough analysis of the various physical regimes is a demanding task.

On the other hand, a few semi-analytical models have been developed in order to capture the essential features of parallel transport in the SOL. In particular, an elegant and exactly-solvable model can be obtained by neglecting the Coulomb force in the parallel kinetic equation [13]. Together with an initial Maxwellian distribution, this surprisingly simple model reproduces with good accuracy some of the main features of an ELM signal, most notably its rapid rise $(\sim 200 \mu \mathrm{s})$ followed by a much slower decay (up to 3ms). The main drawback of this model is that it fails to respect quasineutrality, which is a direct consequence of the Coulomb attraction between the ions and the electrons. (Some form of 'weak' quasi-neutrality can be imposed a posteriori to partially correct this shortcoming [13]).

In the present paper, we adopt an intermediate stance: the fully nonlinear parallel dynamics is solved by means of a self-consistent kinetic model (Vlasov-Poisson equations), but we neglect collisions and effects due to plasma-surface interactions, such as secondary electron emission and recycling. The Vlasov equation is integrated numerically using an Eulerian method (meshing of the entire phase space), which 
guarantees a high resolution in velocity space, even in regions of low plasma density such as the sheaths [14]. In addition, thanks to a recently-developed 'asymptotic-preserving' numerical scheme $[15,16]$, it is possible to lift numerical constraints on the time step and grid spacing, which are no longer limited by, respectively, the electron plasma period and Debye length.

The resulting numerical code is computationally manageable on a desktop computer (2-3 hours of CPU for a typical run, albeit with an effective parallel connection length smaller than its realistic value) and capable of reproducing with good accuracy the principal features of parallel plasma transport during ELMs. The remainder of this paper will be devoted to the description of the model and the analysis of the numerical results for some experimentally-motivated scenarios.

\section{The Vlasov-Poisson approach}

In our approach, we assume that the charged particles (or rather, their guiding centres) travel along the magnetic field lines, but not across them. Thus, we can adopt a onedimensional geometry along the parallel direction, here denoted $x$, with corresponding parallel velocity $v_{x}$. In the perpendicular plane, the distribution function remains Maxwellian at all times, so that the distribution in the four-dimensional phase space $(x, \mathbf{v})$ reads as:

$$
F_{j}(x, \mathbf{v}, t)=f_{j}\left(x, v_{x}, t\right) M_{j}\left(\mathbf{v}_{\perp}\right),
$$

where $M_{j}\left(\mathbf{v}_{\perp}\right)=\left(1 / 2 \pi v_{T j}^{2}\right) \exp \left(-v_{\perp}^{2} / 2 v_{T j}^{2}\right), v_{T j}=\sqrt{T_{j} / m_{j}}$ is the thermal speed, and the label $j=i$, $e$ stands for ions and electrons respectively. The temperature is supposed to be the same for both species, $T_{i}=T_{e}=T_{\mathrm{ELM}}$.

Under these assumptions, the ion and electron evolutions are described by the onedimensional Vlasov equations in the parallel phase space $\left(x, v_{x}\right)$ :

$$
\frac{\partial f_{j}}{\partial t}+v_{x} \frac{\partial f_{j}}{\partial x}-\frac{q_{j}}{m_{j}} \frac{\partial \phi}{\partial x} \frac{\partial f_{j}}{\partial v_{x}}=S_{j}\left(x, v_{x}, t\right)
$$

where $q_{j}= \pm e$ (we consider singly charged ions). The collisionless approximation is reasonable for the initial phase of high-energy transients such as ELMs, for which the thermal mean-free-path exceeds the parallel connection length $L_{\|}$, defined as the typical distance between the outer midplane and the divertor outer target. In the JET tokamak, the parallel connection length is roughly $L_{\|} \sim 30 \mathrm{~m}$.

The source terms $S_{j}$ describe the plasma pulse generated by the ELMs, and can be written as $S_{j}\left(x, v_{x}, t\right)=f_{\mathrm{ELMj}}\left(x, v_{x}\right) g(t)$, where

$$
f_{\mathrm{ELM} j}\left(x, v_{x}\right)=n_{\mathrm{ELM}} \exp \left(-\frac{x^{2}}{2 \sigma^{2}}\right) \frac{\exp \left(-v_{x}^{2} / 2 v_{T j}^{2}\right)}{\sqrt{2 \pi} v_{T j}},
$$

and $g(t)$ models the pulse temporal profile. Thus, the source is a Maxwellian distribution, whose spatial profile is given by a Gaussian with width $\sigma \sim 0.1 L_{\|}$and 
peak density $n_{\text {ELM }}$. Finally, the electrostatic potential $\phi(x, t)$ obeys the one-dimensional Poisson equation

$$
\frac{\partial^{2} \phi}{\partial x^{2}}=-\frac{e}{\epsilon_{0}}\left(n_{i}-n_{e}\right)
$$

The above equations are solved on an interval $x \in\left[-L_{\|}, L_{\|}\right]$, where $x= \pm L_{\|}$represent the locations of the divertor plates. The plates are supposed to be perfectly absorbing surfaces (i.e. the incoming flux is zero) and are kept at constant electric potential, $\phi\left( \pm L_{\|}\right)=0$.

Besides being relevant to the ELM dynamics in a tokamak, the above model describes the general scenario of an initially neutral plasma created in a confined region delimited by two grounded plates: the plasma first undergoes an expansion into vacuum and then reaches the plates. Here, we are mainly interested in the plasma features on the plates, particularly the particles and energy fluxes, defined respectively as (we omit the species index for clarity):

$$
\begin{aligned}
\Gamma(t) & =\iiint v_{x} F\left( \pm L_{\|}, \mathbf{v}, t\right) d \mathbf{v} \\
Q(t) & =\iiint \frac{1}{2} m\left(v_{x}^{2}+v_{\perp}^{2}\right) v_{x} F\left( \pm L_{\|}, \mathbf{v}, t\right) d \mathbf{v}=Q_{\|}+Q_{\perp} .
\end{aligned}
$$

It can be readily checked that $Q_{\perp}=\Gamma T_{\mathrm{ELM}}$.

We use typical parameters for Type-I ELMs [13]: plasma density $n_{\text {ELM }}=5 \times$ $10^{19} \mathrm{~m}^{-3}$, temperature $T_{\text {ELM }}=1.5 \mathrm{keV}$, and parallel connection length $L_{\|}=30 \mathrm{~m}$. With these values (and considering hydrogen ions, $m_{i}=1836 m_{e}$ ) the ion traversal time is $\tau_{\| i}=L_{\|} / v_{T i} \sim 80 \mu \mathrm{s}$ and the Debye length $\lambda_{D} \sim 40 \mu \mathrm{m}$. In the simulation results, the parallel energy fluxes will be expressed in terms of $Q_{\text {ref }}=n_{\mathrm{ELM}} v_{T i} T_{\mathrm{ELM}}$, which, for the above set of parameters, is $Q_{\text {ref }}=4.5 \mathrm{GWm}^{-2}$.

The relevant physical regime will be determined by two dimensionless parameters, namely, the ion-to-electron mass ratio and the ratio of the Debye length to the parallel connection length, $\lambda \equiv \lambda_{D} / L_{\|} \sim 10^{-6}$. The latter is particularly important as it determines the scale length over which quasi-neutrality can be violated. This can be easily seen by rewriting Poisson's equation in dimensionless units, normalizing space to $L_{\|}$, the densities to $n_{\mathrm{ELM}}$, and the electric potential to $T_{\mathrm{ELM}} / e$ :

$$
\lambda^{2} \frac{\partial^{2} \phi}{\partial x^{2}}=n_{e}-n_{i}
$$

When $\lambda \rightarrow 0$, the only way to satisfy Poisson's equation is for the plasma to be quasineutral $\left(n_{e}-n_{i} \rightarrow 0\right)$. The electric potential does not vanish, but takes exactly the value required to enforce the quasi-neutrality constraint. In that case, Poisson's equation cannot be used to determine the potential, as Eq. (7) becomes singular.

When $\lambda$ is finite but very small, as is the case for our problem, quasi-neutrality is satisfied over distances much larger than the Debye length, but breaks down at finer scales (a typical instance are the sheaths that form at the plasma-surface boundary, which have a thickness of a few Debye lengths). Thus, an increasingly fine spatial 
resolution is required as $\lambda \rightarrow 0$. In addition, since the plasma frequency scales as $\lambda^{-1}$, the time step must also be reduced in the same proportions. Indeed, it has been proven that a numerical instability occurs if the adopted time step is larger than the inverse plasma frequency [15].

For the above reasons, it would be useful to replace the standard Poisson's equation (7) with an equivalent equation that can provide $\phi$ when $\lambda \rightarrow 0$. This can actually be achieved rather easily $[15,16]$ by using the mass and momentum conservation equations derived from the Vlasov equations (2). The resulting 'reformulated' Poisson's equation, written in appropriate dimensionless units, reads as:

$$
\lambda^{2} \frac{\partial^{2}}{\partial t^{2}}\left(\frac{\partial^{2} \phi}{\partial x^{2}}\right)+\frac{\partial}{\partial x}\left[\left(\varepsilon n_{i}+n_{e}\right) \frac{\partial \phi}{\partial x}\right]=-\frac{\partial^{2}}{\partial x^{2}}\left(R_{i}-R_{e}\right),
$$

where $\varepsilon=m_{e} / m_{i}$ and $R_{j}=\int f_{j} v^{2} d v$ (see the Appendix A for details on its derivation). Equation (8) is completely equivalent to the standard Poisson equation (provided the latter and its time derivative are satisfied at $t=0$ ), but does not become singular in the asymptotic regime $\lambda \rightarrow 0$. For this reason, the above approach has been termed 'asymptotic-preserving' method.

Thanks to the asymptotic-preserving scheme, it is possible to employ a grid spacing that exceeds the Debye length and, more importantly, a time step larger than the inverse plasma frequency. In the forthcoming simulations, we have used a grid spacing $\Delta x=2 \lambda_{D}$ and a time step up to $\Delta t=4 \omega_{p e}$. Note that here $\lambda_{D}$ and $\omega_{p e}$ refer to their initial values computed with the peak density $n_{\text {ELM }}$ and temperature $T_{\text {ELM }}$. These values, in the normalisation of Eq. (7), are equal to $\lambda$ and $\lambda^{-1}$, respectively.

Some details on the numerical techniques used to solve the model equations are provided in Appendix B.

\section{Free-streaming model}

Fundamenski et al. [13] have developed a simple analytical model for parallel transport, which nevertheless captures the main features of an ELM signal, most notably its rapid rise and slower decay. The model completely neglects Coulomb interactions, so that the Vlasov equations are reduced to the following free-streaming equations (in the rest of this section we omit the species index for simplicity):

$$
\frac{\partial f}{\partial t}+v_{x} \frac{\partial f}{\partial x}=S\left(x, v_{x}, t\right)
$$

This equation possesses the exact solution

$$
f\left(x, v_{x}, t\right)=\int_{0}^{t} f_{\mathrm{ELM}}\left(x-v_{x} t+v_{x} t^{\prime}, v\right) g\left(t^{\prime}\right) d t^{\prime} .
$$

We further assume that the source is instantaneous in time, $g(t)=\delta(t)$, where $\delta$ is the Dirac delta function, and that it is localized in space, $n_{\mathrm{ELM}}(x) \equiv \int_{-\infty}^{\infty} f_{\mathrm{ELM}}(x, v) d v=$ $\sqrt{2 \pi} \sigma \delta(x)$. With these hypotheses, it is possible to express analytically all relevant quantities at an arbitrary location $x$. 
For the particles density, we obtain:

$$
N(x, t) \equiv \iiint F(x, \mathbf{v}, t) d \mathbf{v}=\frac{\sigma}{v_{T}} \frac{n_{\mathrm{ELM}}}{t} \exp \left(-\frac{x^{2}}{2 t^{2} v_{T}^{2}}\right),
$$

whereas the particles flux yields: $\Gamma=(x / t) N$. Further, we have for the kinetic energy:

$$
\mathcal{E}(x, t) \equiv \frac{1}{N} \iiint F(x, \mathbf{v}, t) \frac{m}{2} \mathbf{v}^{2} d \mathbf{v}=\mathcal{E}_{\|}+\mathcal{E}_{\perp}=\frac{m}{2} \frac{x^{2}}{t^{2}}+T_{\mathrm{ELM}}
$$

and the energy flux:

$$
Q(x, t)=Q_{\|}+Q_{\perp}=\frac{m}{2} \frac{x^{2}}{t^{2}} \Gamma+\Gamma T_{\mathrm{ELM}}
$$

Using these expressions, we can define the parallel sheath transmission coefficient: $\gamma_{\|}=Q_{\|} /\left(\Gamma \mathcal{E}_{\|}\right)$, which is exactly equal to unity within this model.

The above formulae will be used as a benchmark for the numerical results presented in the next section.

\section{Simulation results}

We now present the numerical results obtained by solving the full Vlasov-Poisson equations, i.e. including the effect of the electric field. We shall use two types of temporal profiles for the sources in the Vlasov equations (2): an instantaneous source $g(t)=\delta(t)$, and a distributed-in-time source with

$$
g(t)=C t^{2} \exp \left(-\frac{\left(t-t_{0}\right)^{2}}{2 \sigma_{t}^{2}}\right)
$$

where the normalization constant $C$ is chosen so that $\int_{0}^{\infty} g(t) d t=1$. Provided that $\sigma_{t} \ll t_{0}$, the above expression peaks at $t=\left(t_{0} / 2\right)\left(1+\sqrt{1+8 \sigma_{t}^{2} / t_{0}^{2}}\right)$ and has the advantage of vanishing at $t=0$.

In all cases, except where otherwise stated, we use the following parameters: $m_{i}=1836 m_{e}, \sigma_{i}=0.1 L_{\|}$, and $\lambda=10^{-3}$. This value of $\lambda$ is much larger than the realistic value for ELM plasmas. However, we have checked that the evolution of relevant quantities (such as the particles and energy fluxes) are virtually unchanged when smaller values of $\lambda$ are taken (an exception is an early peak in the electron energy flux, which disappears in the asymptotic limit $\lambda \rightarrow 0$; see later for further details). Thus, it appears that for $\lambda=10^{-3}$ we have already reached the asymptotic regime and the results can be confidently extrapolated to the realistic value of $\lambda$.

\subsection{Instantaneous source}

First of all, we tested our numerical code by comparing the analytical results from the free-streaming model with the numerical solution of the Vlasov equation without electric field (9). As expected, the analytical and numerical fluxes (not shown here) were virtually identical.

Next, we turn to the full solution of the Vlasov-Poisson equations including the electric field. Figure 1 shows the evolution of the electron and ion particles and energy 

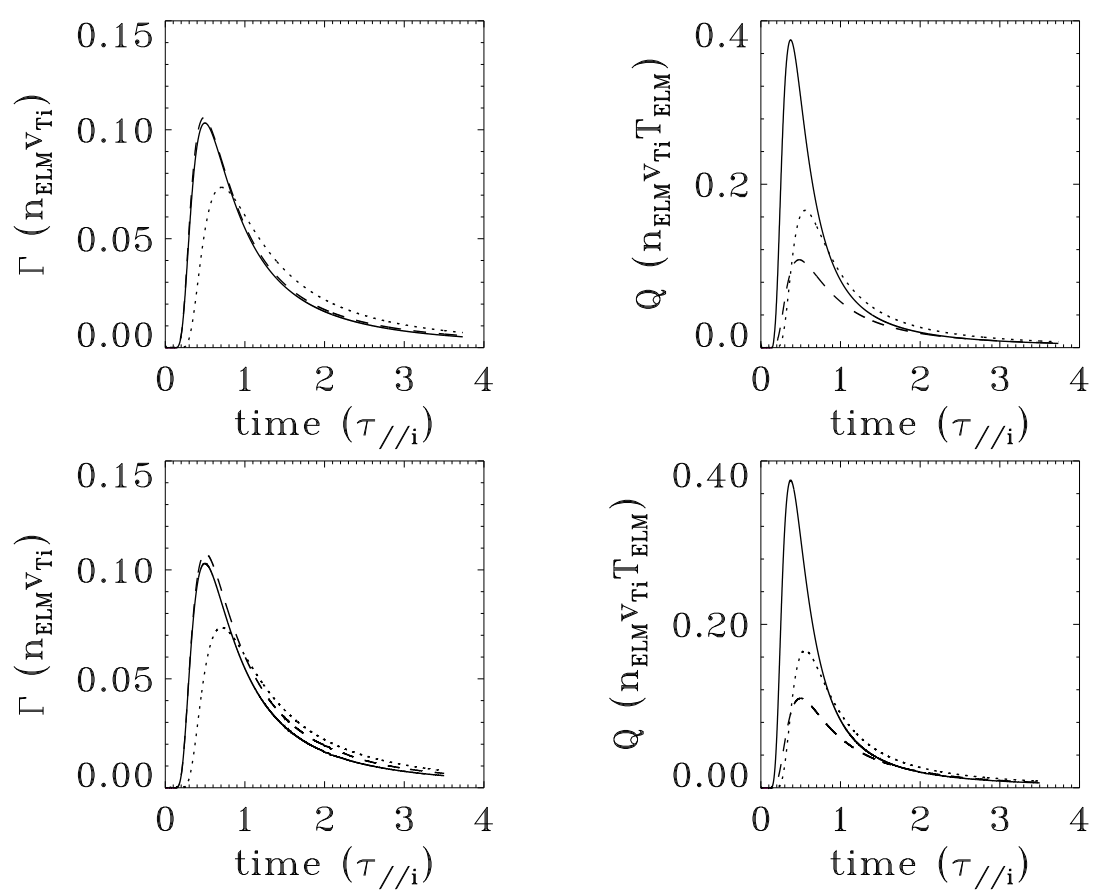

Figure 1. Particles (left panel) and energy (right panel) fluxes as a function of time normalized to the ion transit time. Solid lines refer to the ions, dashed lines to the electrons. The dotted lines represent the ion fluxes obtained from the free-streaming model. Top panels: $\lambda=10^{-3}$; bottom panels: $\lambda=2.5 \times 10^{-4}$.

fluxes on the divertor plates (since this case is symmetric there is no difference between the inner and the outer plate), for $\lambda=10^{-3}$ and $\lambda=2.5 \times 10^{-4}$. The standard pattern (sharp rise, followed by a slower decay) is recovered in the simulations.

Very little difference in the fluxes is observed between the $\lambda=10^{-3}$ and the $\lambda=2.5 \times 10^{-4}$ cases. Therefore, it seems that the asymptotic regime $(\lambda \rightarrow 0)$ has already been reached, so that the results for the fluxes should remain unchanged for a realistic value of $\lambda$, which is of the order $10^{-6}$.

The ion and electron particles fluxes are virtually identical, which is a consequence of quasi-neutrality. In contrast, the electron energy flux is appreciably smaller than that of the ions. This is in line with measurements of the heat load on a tokamak divertor plates, where the electron load is observed to be roughly one third of the ion load $[17,18]$. The free-streaming model (dotted line in Fig. 1) is fairly accurate for the ion fluxes, especially over long times.

The above dynamics occurs on the timescale of the ionic transit time $\tau_{\| i}$, as is expected from quasi-neutrality. However, an early burst of electrons is observed on the electronic transit time scale, as can be seen from Fig. 2. This burst is due to a bunch of electrons escaping the attraction of the ions and reaching the plates before quasineutrality can be established. Indeed, the height of this peak decreases with decreasing $\lambda$, i.e. when quasi-neutrality is stronger. From the free-streaming result [Eq. (13)], the electron energy flux should peak at $t_{\text {peak }}=L / 2 v_{T e} \sim 0.5 \tau_{\| e}$. The observed peak 


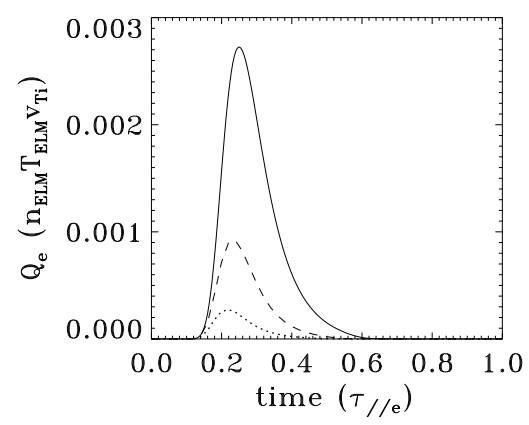

Figure 2. Electron energy flux on the divertor plate for short times, for three values of the normalized Debye length: $\lambda=10^{-3}$ (solid line), $\lambda=5 \times 10^{-4}$ (dashed line), and $\lambda=2.5 \times 10^{-4}$ (dotted line). Note that here time is normalized to the electron transit time $\tau_{\| e}=\left(m_{e} / m_{i}\right) \tau_{\| i}$.



Figure 3. Diamonds: Electron kinetic energy deposited on the right plate $\left(x=L_{\|}\right)$ as a function of $\lambda$. The straight solid ine represents the curve $E_{\text {elec }} / T_{\mathrm{ELM}}=15 \lambda^{2}$.

is at roughly one half of this value, signalling that the peak is caused by suprathermal electrons travelling with an average velocity equal to twice the thermal speed.

It would be interesting to deduce a scaling law for the deposited electron energy as a function of $\lambda$. This can be done by integrating the electron fluxes on Fig. 2 between $t=0$ and, say, $t=\tau_{\| e}$. The electron kinetic energy deposited on the divertor plate $\left(x=L_{\|}\right)$is then defined as $E_{\text {elec }}=\int_{0}^{\tau_{\| e}} Q_{e}(t) d t$ and is shown in Fig. 3 on a log-log plot. It is clear that $E_{\text {elec }}$ depends quadratically on $\lambda$. In the present case, we obtain a scaling law of the type: $E_{\text {elec }} / T_{\mathrm{ELM}} \approx 15 \lambda^{2}$, which allows us to extrapolate the result to realistic values of $\lambda$.

The mechanism responsible for the above effect is best understood by inspecting the parallel phase space $\left(x, v_{x}\right)$, displayed in Fig. 4, where we show the contour plots of the electron distribution function at various times. The electron velocity distribution on the right boundary is shown on Fig. 5 at the same instants. Very early, a small fraction (about $0.2 \%$ ) of energetic electrons is ejected from the bulk plasma and hits the divertor plate. This leaves behind a net positive charge that traps the remaining electrons in a potential well, preventing them to reach the divertor plates. The potential well expands slowly following the motion of the ions (on a timescale $\sim \tau_{\| i}$ ) and finally 

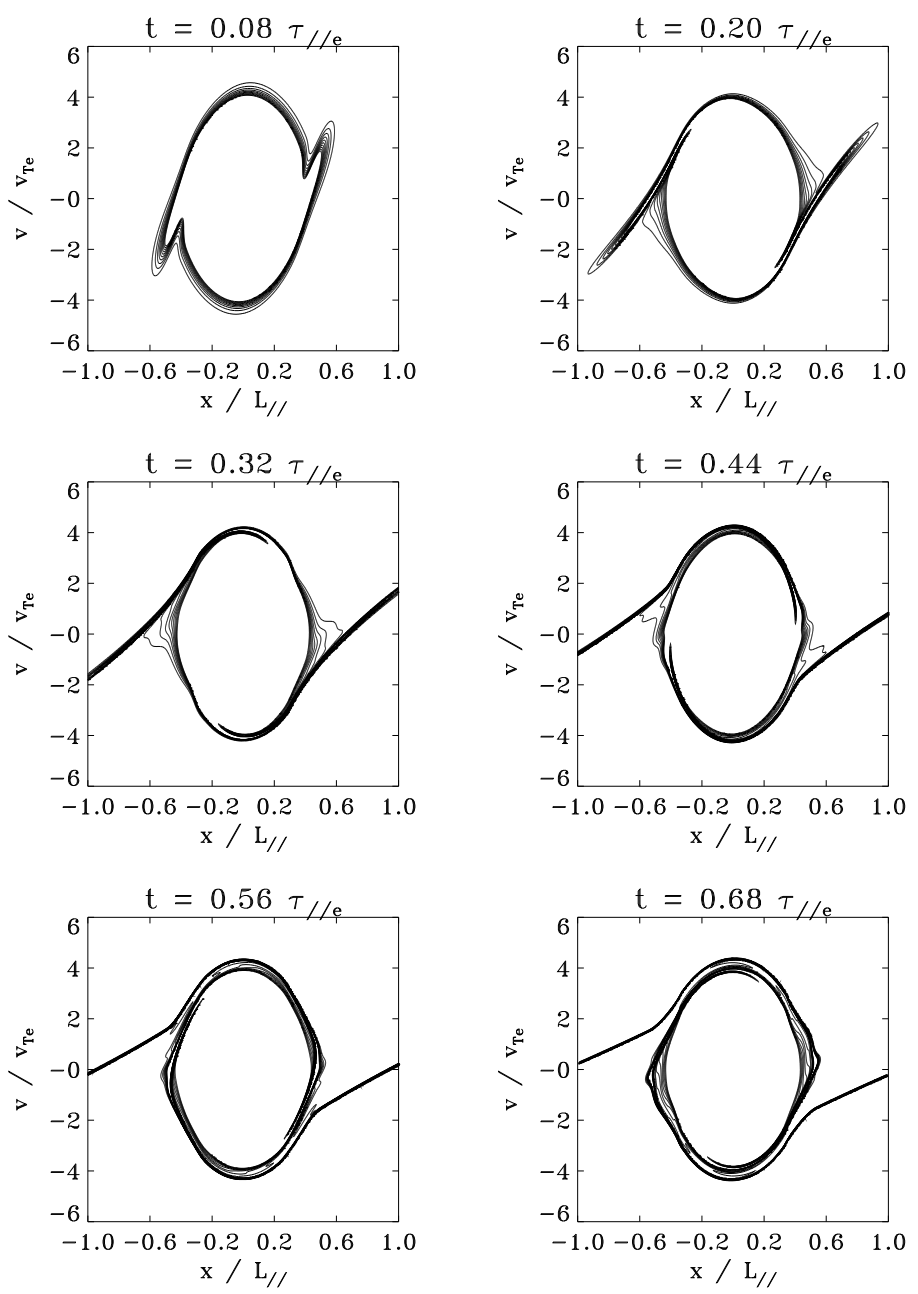

Figure 4. Contour plots of the electron distribution function in the parallel phase space at different times, measured in units of the electron transit time $\tau_{\| e}=$ $\left(m_{e} / m_{i}\right) \tau_{\| i}$. Only contour levels for which $f_{e} \leq 10^{-4}$ are shown.

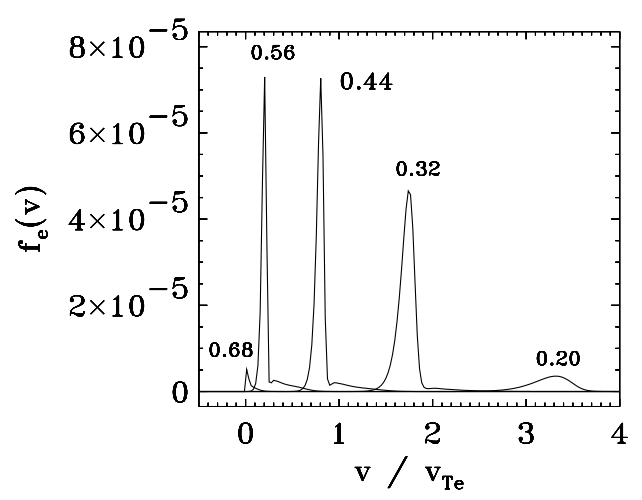

Figure 5. Electron parallel velocity distribution at $x=L_{\|}$at different times, measured in units of the electron transit time $\tau_{\| e}=\left(m_{e} / m_{i}\right) \tau_{\| i}$. 

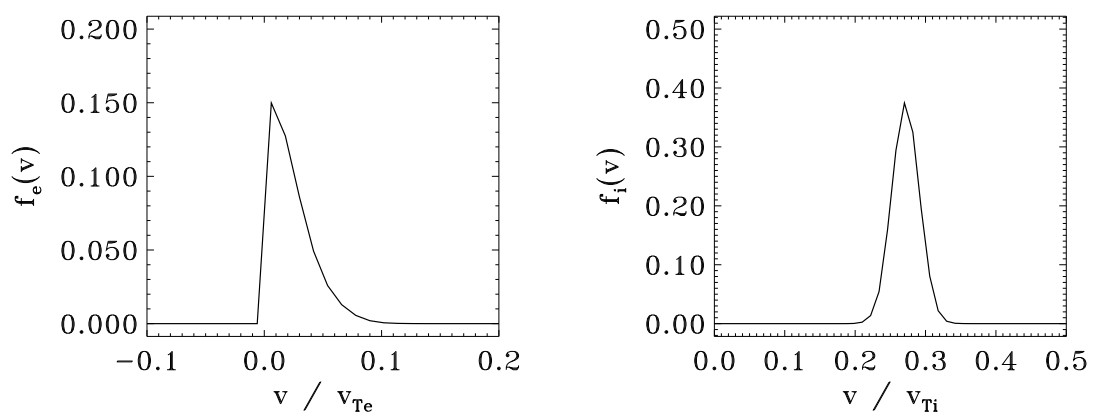

Figure 6. Electron (left panel) and ion (right panel) velocity distribution at $x=L_{\|}$ at the end of the run $\left(t=3.73 \tau_{\| i}\right)$.
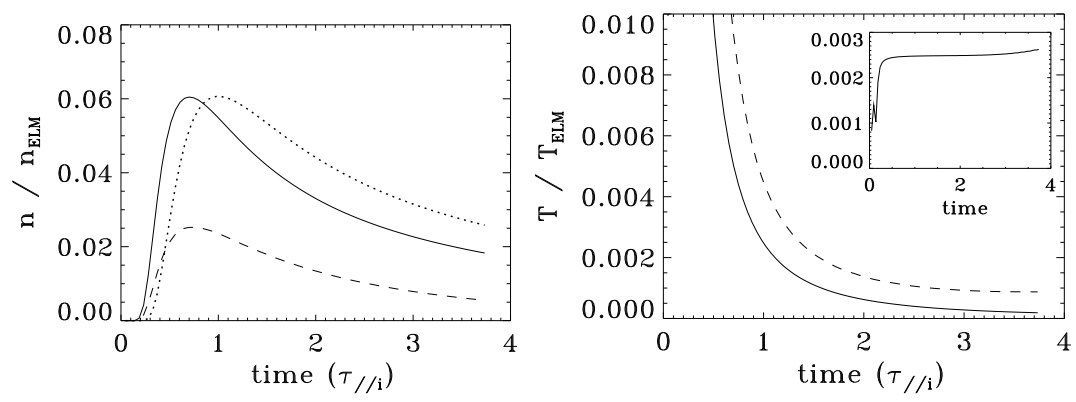

Figure 7. Time evolution of densities (left panel) and parallel temperatures (right panel) at $x=L_{\|}$. The temperatures are normalized to their initial value $T_{\text {ELM }}$. Solid line: ions; dashed line: electrons. The dotted line in the left panel represents the theoretical prediction of Eq. (11) for the ions. The inset in the right panel shows the ion temperature multiplied by $t^{2}$.

reaches the boundary, thus releasing the ions' and the (remaining) electrons' energy onto the divertor plate. On the timescale of the ion dynamics, the velocity distributions on the wall become approximately half-Maxwellian for the electrons and Maxwellian for the ions [19], as can be seen from Fig. 6 .

The densities and temperatures of the two species of particles on the plate are depicted in Fig. 7. The temperatures are particularly interesting: whereas the electron temperature converges to a constant (about one thousandth of the initial value $T_{\mathrm{ELM}}$ ), the ion temperature decreases with time as $t^{-2}$. This type of decrease is readily obtained from the free-streaming model and was anticipated in earlier studies [20]. (One should keep in mind that here we are talking about the parallel temperatures; the perpendicular temperatures remain constant for all times, since no coupling between the parallel and perpendicular motion is allowed in our model).

The parallel sheath transmission coefficients are shown in Fig. 8. Whereas $\gamma_{\| i}$ quickly reaches unity, as expected from the free-streaming model, $\gamma_{\| e}$ is twice as large. This is due to the fact that the electronic distribution on the divertor plate is a halfMaxwellian (see Fig. 6), so that the parallel kinetic energy $\mathcal{E}_{\|}$is twice as small compared to a full Maxwellian. 


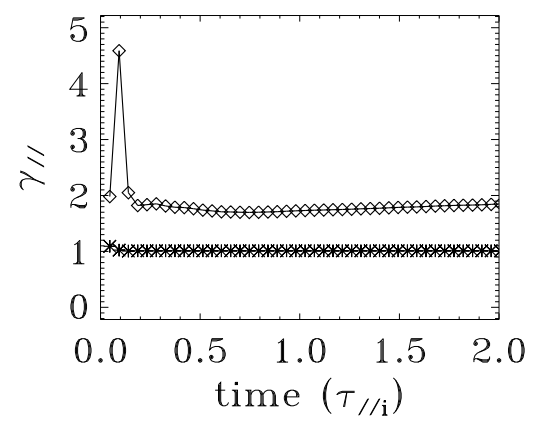

Figure 8. Parallel sheath transmission coefficients for the electrons (diamonds) and the ions (stars).

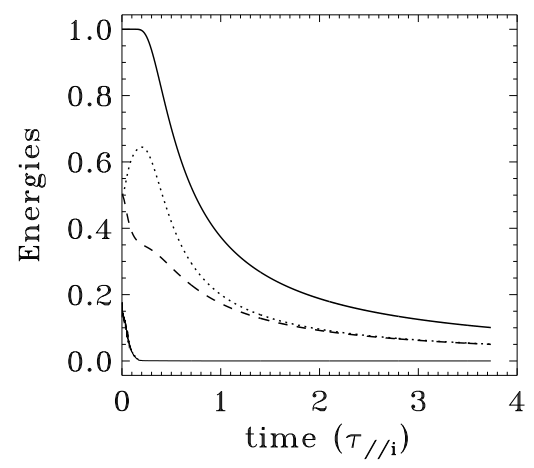

Figure 9. Time evolution of the total ion kinetic energy (dotted line), electron kinetic energy (dashed line), potential energy $(\times 1000)$ (thin solid line), and total energy (thick solid line). All quantities are normalized to the initial total energy.

Finally, we show the time evolution of some integrated quantities, particularly the kinetic and potential energies (Fig. 9). As long as the main plasma population does not reaches the plates, it expands freely into a vacuum. During this phase, lasting up to $\sim 0.2 \tau_{\| i}$, some kinetic energy is transferred from the electrons to the ions.

This transfer occurs because of the fast electrons that move rapidly forwards leaving the ions behind, as was shown in Figs. 4-5. This creates a charge imbalance - and therefore an electric field - at the plasma-vacuum front, which is revealed by the peak of the potential energy in Fig. 9. The electric field slows down the electrons and accelerates the ions, thus effectively transferring some kinetic energy from the former to the latter. Eventually, the ions catch up with the electrons, the plasma becomes locally neutral, and the potential energy goes to zero. Over longer times $\left(t>0.2 \tau_{\| i}\right)$, the charged particles start being lost on the divertor plates and consequently both kinetic energies decrease.

\subsection{Time-distributed source}

In actual ELM events, the plasma pulse is not instantaneous, but has a finite duration, which is of the order of a few ion transit times. In order to simulate this type of scenario, 

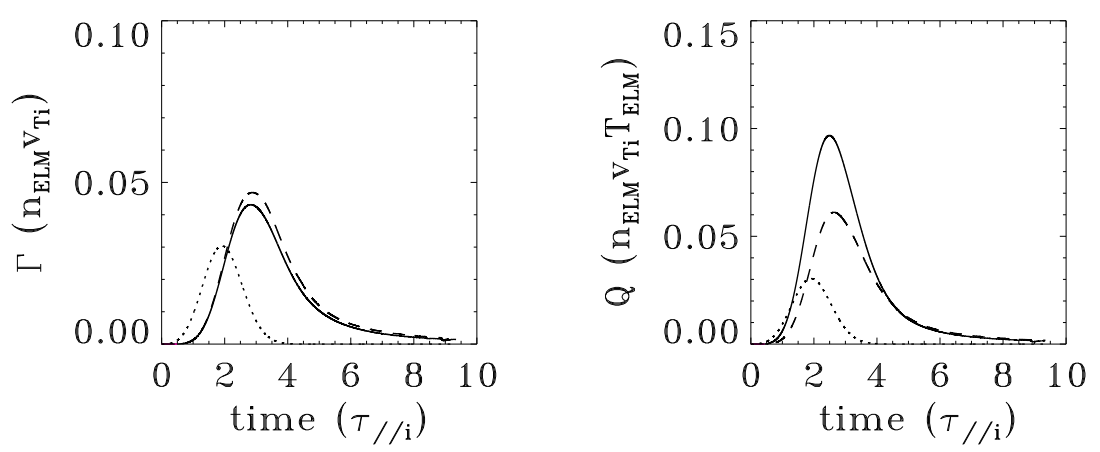

Figure 10. Particles (left panel) and energy (right panel) fluxes as a function of time, for a case with time-distributed source. Solid lines refer to the ions, dashed lines to the electrons. The dotted lines represent the source temporal profile $g(t)$, in arbitrary units.


Figure 11. Same as Fig. 10 for free-streaming particles (no electric field).

we use a pulse temporal profile such as that of Eq. (14), with $\sigma_{t}=0.7 \tau_{\| i}$ and $t_{0}=1.4 \tau_{\| i}$ [the plot of $g(t)$, given by the dotted line on Fig. 10, shows that the pulse peaks at about $\left.1.9 \tau_{\| i}\right]$.

The evolution of the particles and energy fluxes is shown in Fig. 10. The fluxes reach a maximum at roughly one ion transit time after the source peak. The ion energy flux is about $30 \%$ larger than that of the electrons, whereas it was almost four times larger in the case of an instantaneous source (Fig. 1). It is useful to compare these results to a free-streaming case, for which the electric field was neglected (Fig. 11). In the free-streaming case, the electron fluxes peak earlier, basically in phase with the source (at closer inspection, the electron fluxes peak around 2-3 electron transit times after the peak of the source). Also, a larger fraction of the energy flux is carried by the electrons, at the expense of the ions' energy flux. Clearly, the electric field has the effect of transferring some energy from the electrons to the ions.

By inspecting the electron energy flux for short times (Fig. 12), we also observe a small peak occurring on the electronic timescale, as we had found for the case of an instantaneous source. Now the peak appears later, since the plasma needs to build up progressively, but still occurs much earlier than the peak of the ELM pulse (see inset of 




Figure 12. Electron energy flux on the divertor plate for short times (in units of the electron transit time). The inset shows the temporal profile of the source $g(t)$.
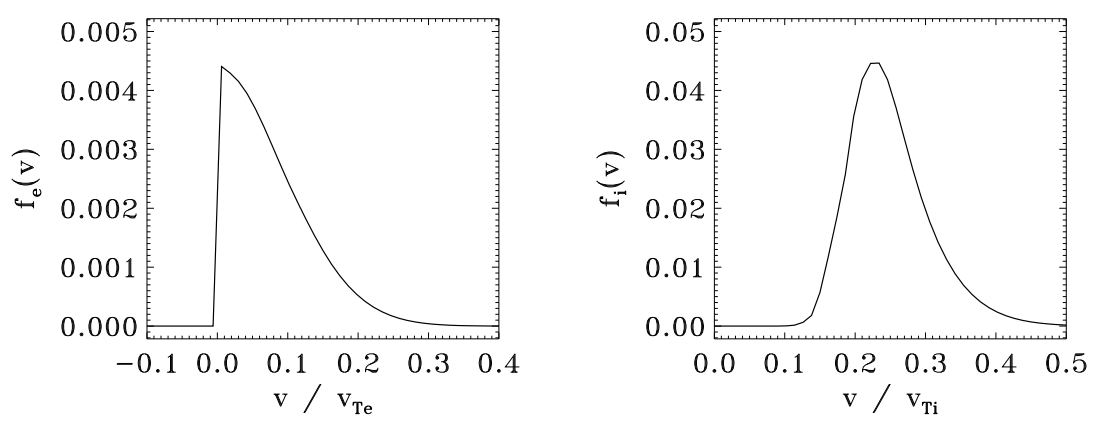

Figure 13. Electron (left panel) and ion (right panel) velocity distribution at $x=L_{\|}$ at the end of the run $\left(t=9.34 \tau_{\| i}\right)$, for a case with time-distributed source.

Fig. 12).

The velocity profiles on the divertor plate (Fig. 13) are similar to those obtained in the case of an instantaneous source, i.e. half-Maxwellian for the electrons and roughly Maxwellian for the ions.

Finally, the parallel sheath transmission coefficients are shown in Fig. 14, revealing some interesting behaviour. The ion coefficient remains close to unity for the entire duration of the run, signalling that the Vlasov-Poisson result stays close to the free-streaming solution. The electronic coefficient displays a long transient, which corresponds to the time-window when the source is active, then quickly relaxes to its asymptotic value $\left(\gamma_{\| e}=2\right)$ after the source is extinguished.

\section{Conclusion}

Parallel transport plays a major role in the dynamics of ELM events in a tokamak. Even relatively simple models that ignore Coulomb interactions [13] manage to reproduce with fair accuracy the main features of an ELM event, particularly the energy load on the divertor plates.

Stimulated by this observation, we constructed a minimal kinetic model of parallel 


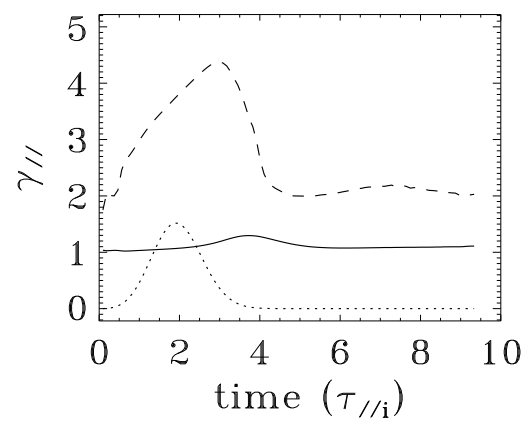

Figure 14. Parallel sheath transmission coefficients for the ions (solid line) and the electrons (dashed line), for a case with time-distributed source. The dotted lines represent the source temporal profile $g(t)$, in arbitrary units.

transport during ELMs, based on the Vlasov-Poisson equations in the direction parallel to the magnetic field (in the perpendicular plane, the plasma is supposed to be Maxwellian). The results of numerical simulations performed with this model were compared systematically to the free-streaming solutions [13].

Several interesting conclusions can be drawn from our study:

(i) Even for moderate connection lengths (we used $L_{\|}=1000 \lambda_{D}$ throughout this work) the particles and energy fluxes are correctly described, i.e. by decreasing the ratio $\lambda=\lambda_{D} / L_{\|}$the fluxes are virtually unchanged;

(ii) Whereas the ion fluxes are relatively well reproduced by the free-streaming model, the electron fluxes are not. This is because the free-streaming model does not properly include quasi-neutrality;

(iii) On the electron transit time scale, an early burst can be observed on the divertor plates, which corresponds to suprathermal electrons escaping the ions' attraction due to their large kinetic energy. The remaining electrons are trapped in the potential well created by the ions (which now outnumber the electrons) and evolve in unison with them. On the ion timescale, the plasma is everywhere neutral, except for a positive net charge in front of the divertor plates (sheaths);

(iv) The long-time velocity distributions on the plates are Maxwellian for the ions and half-Maxwellian for the electrons;

(v) The parallel sheath transmission coefficients tend rapidly to 1 for the ions and 2 for the electrons;

(vi) Most of the above results hold equally for an ELM pulse with a realistic duration $t_{\mathrm{ELM}} \sim \tau_{\| i}$.

The present model could be further improved in several ways. First, a pre-ELM environment (low temperatures and densities) should be computed self-consistently and used as a starting background prior to the ELM pulse. Second, even though collisions are not dominant due to the high temperature of the ELM pulse, they may play a role over long times, so that a suitable collision term should be added to the Vlasov equations. 
Finally, the perpendicular dynamics should be treated with more sophistication, by taking into account that the magnetic field decreases with increasing distance from the tokamak axis. This introduces a 'mirror' force that acts differently on ions with different magnetic moments (this effect should be negligible for the electrons) and couples the parallel and perpendicular dynamics. A sufficiently accurate description could be achieved by assuming that the magnetic moment is an exact invariant and then using an approach such as that of Ref. [21]. The above issues are currently under investigation.

\section{Acknowledgments}

We thank W. Fundamenski for several useful discussions.

\section{Appendix A. Reformulated Poisson's equation}

As mentioned in Section 2, in order to compute the potential $\phi$ leading to a quasineutral regime (the limit $\lambda \rightarrow 0$ ) we need a reformulation of the Poisson's equation that does not become singular in this limit. Such an equation was already derived in the case of the Euler-Poisson [15] and the Vlasov-Poisson systems [22, 16]. For the sake of completeness, we show how the reformulated Vlasov-Poisson model is obtained in our case.

We start with the following scaled Vlasov-Poisson equations

$$
\begin{aligned}
& \frac{\partial f_{i}}{\partial t}+v_{x} \frac{\partial f_{i}}{\partial x}-\varepsilon \frac{\partial \phi}{\partial x} \frac{\partial f_{i}}{\partial v_{x}}=S_{i}\left(x, v_{x}, t\right) \\
& \frac{\partial f_{e}}{\partial t}+v_{x} \frac{\partial f_{e}}{\partial x}+\frac{\partial \phi}{\partial x} \frac{\partial f_{e}}{\partial v_{x}}=S_{e}\left(x, v_{x}, t\right) \\
& -\lambda^{2} \frac{\partial^{2} \phi}{\partial x^{2}}=n_{i}-n_{e},
\end{aligned}
$$

which are obtained by normalizing Eqs. (2)-(4) as follows

$$
\widehat{x}=\frac{x}{L_{\|}} \quad \widehat{v}_{x}=\frac{v_{x}}{v_{T_{e}}} \quad \widehat{t}=\frac{v_{T_{e}}}{L_{\|}} t \quad \widehat{n}_{j}=\frac{n_{j}}{n_{\mathrm{ELM}}} \quad \widehat{\phi}=\frac{\phi}{T_{e} / e}
$$

(the overcarets, which will be omitted in the sequel, stand for the dimensionless variables). By taking the first two moments of the difference between Eq. (A.1) and Eq. (A.2), we obtain

$$
\begin{aligned}
& \partial_{t}\left(n_{i}-n_{e}\right)+\partial_{x}\left(J_{i}-J_{e}\right)=0 \\
& \partial_{t}\left(J_{i}-J_{e}\right)+\partial_{x}\left(R_{i}-R_{e}\right)+\left(\varepsilon n_{i}+n_{e}\right) \partial_{x} \phi=0,
\end{aligned}
$$

where $J_{j}=\int f_{j}\left(x, v_{x}, t\right) v_{x} d v_{x}$ and $R_{j}=\int f_{j}\left(x, v_{x}, t\right) v_{x}^{2} d v_{x}$. Now we subtract the spatial derivative of Eq. (A.6) from the time derivative of Eq. (A.5):

$$
\frac{\partial^{2}}{\partial t^{2}}\left(n_{i}-n_{e}\right)-\frac{\partial}{\partial x}\left[\left(\varepsilon n_{i}+n_{e}\right) \frac{\partial \phi}{\partial x}\right]=\frac{\partial^{2}}{\partial x^{2}}\left(R_{i}-R_{e}\right) .
$$

Finally, using Poisson's equation we express $\partial_{t t}^{2}\left(n_{i}-n_{e}\right)$ in terms of $\phi$ and thus recover the reformulated Poisson equation (8). 
It can further be shown [16, 22] that the reformulated Poisson equation (8) (coupled to the Vlasov equation) is rigorously equivalent to the standard Poisson equation (7) provided that $\phi$ satisfy the following conditions at the initial time: $\left(\lambda^{2} \partial_{x x}^{2} \phi+n_{i}-n_{e}\right)_{t=0}=$ 0 and $\left(\frac{\partial}{\partial t}\left(\lambda^{2} \partial_{x x}^{2} \phi+n_{i}-n_{e}\right)\right)_{t=0}=0$.

\section{Appendix B. Numerical methods}

The Vlasov equation (2) is solved using an Eulerian method based on a uniform meshing of the parallel phase space $\left(x, v_{x}\right)$. For the time-stepping, a second-order splitting scheme is used [14], which solves alternatively the advection in real space, $\partial_{t} f+v_{x} \partial_{x} f=0$, and the advection in velocity space, $\partial_{t} f+(q E / m) \partial_{v_{x}} f=0$. Each advection step is performed using a third-order positive flux-conservative (PFC) method, with a slope corrector that prevents the distribution function from becoming negative [23, 24]. A typical simulation requires 1000 points both in real space and in velocity space.

As to the reformulated Poisson's equation, it was shown that an explicit timestepping scheme is only stable if $\Delta t \leq \omega_{p e}^{-1} \sim \lambda[15]$. In order to lift this numerical constraint, the reformulated Poisson equation must be solved through an implicit scheme. More precisely, we first discretize Eq. (8) as follows:

$$
-\frac{\partial}{\partial x}\left(\lambda^{2} \frac{\partial_{x} \phi^{m+1}-2 \partial_{x} \phi^{m}+\partial_{x} \phi^{m-1}}{\Delta t^{2}}+\left(\varepsilon n_{i}^{m}+n_{e}^{m}\right) \partial_{x} \phi^{m+1}\right)=\frac{\partial^{2}}{\partial x^{2}}\left(R_{i}^{m}-R_{e}^{m}\right) .
$$

Then, using Poisson's equation, we replace $\partial_{x x}^{2} \phi^{m}$ and $\partial_{x x}^{2} \phi^{m-1}$ by density terms at the corresponding time steps:

$$
\begin{aligned}
-\frac{\partial}{\partial x} & {\left[\left(\frac{\lambda^{2}}{\Delta t^{2}}+\varepsilon n_{i}^{m}+n_{e}^{m}\right) \partial_{x} \phi^{m+1}\right] } \\
& =\frac{\partial^{2}}{\partial x^{2}}\left(R_{i}^{m}-R_{e}^{m}\right)+\frac{1}{\Delta t^{2}}\left(2\left(n_{i}^{m}-n_{e}^{m}\right)-\left(n_{i}^{m-1}-n_{e}^{m-1}\right)\right) .
\end{aligned}
$$

Next, we make use of a discrete form of Eq. (A.5) to replace $\left(n_{i}^{m}-n_{e}^{m}\right)-\left(n_{i}^{m-1}-n_{e}^{m-1}\right)$ with $-\Delta t\left(\partial_{x} J_{i}^{m}-\partial_{x} J_{e}^{m}\right)$. We thus obtain the discrete (in the time variable) version of the reformulated Poisson's equation

$$
\begin{aligned}
-\frac{\partial}{\partial x} & {\left[\left(\lambda^{2}+\Delta t^{2}\left(\varepsilon n_{i}^{m}+n_{e}^{m}\right)\right) \partial_{x} \phi^{m+1}\right] } \\
& =\Delta t^{2} \frac{\partial^{2}}{\partial x^{2}}\left(R_{i}^{m}-R_{e}^{m}\right)+\left(n_{i}^{m}-n_{e}^{m}\right)-\Delta t\left(\partial_{x} J_{i}^{m}-\partial_{x} J_{e}^{m}\right),
\end{aligned}
$$

which allows us to compute $\phi^{m+1}$ from known quantities at time $t^{m}[16]$. A finite difference method is then used to approximate the spatial derivatives in the equation.

Although the above time-stepping strategy is only first order in time, the method could be easily extended to second order accuracy. Some work along this direction was presented in Ref. [15].

Finally, we remark that: (i) when $\Delta t \rightarrow 0$ for fixed $\lambda$, Eq. (B.1) reduces to a discrete form of the standard Poisson's equation; (ii) when $\lambda \rightarrow 0$ for fixed $\Delta t$, the scheme leads to a consistent (non-singular) discretization of the $\lambda \rightarrow 0$ limit of Eq. 
(8). Indeed, the last two terms in Eq. (B.1) are of order $O\left(\lambda^{2}\right)$ in virtue of Poisson's equation, and therefore vanish when $\lambda \rightarrow 0$.

\section{References}

[1] G. Janeschitz, ITER JCT and HTs, J. Nucl. Mater. 290-293, 1 (2001).

[2] R. Behrisch, G. Federici, A. Kukushkin, and D. Reiter, J. Nucl. Mater. 313-316, 388 (2003).

[3] P. C. Stangeby, The plasma boundary of magnetic fusion devices (Institute of Physics Publishing, London, 2000).

[4] R. Chodura, Phys. Fluids 25, 1628 (1982).

[5] S. Devaux and G. Manfredi, Phys. Plasmas 13, 083504, (2006).

[6] S. Devaux and G. Manfredi, Plasma Phys. Control. Fusion 50, 025009 (2008).

[7] E. Ahedo, Phys. Plasmas 4, 4419 (1997).

[8] B. Lipschultz et al., Nucl. Fusion 47, 1189 (2007).

[9] M. Bécoulet et al., J. Nuc. Mater. 337-339, 677 (2005).

[10] R.A. Pitts, W. Fundamenski, S.K. Erents, Y. Andrew, A. Loarte, C. Silva, and JET EFDA contributors, Nucl. Fusion 46, 82 (2006).

[11] D. Tskhakaya, F. Subba, X. Bonnin, D.P. Coster, W. Fundamenski, R.A. Pitts, and JET EFDA contributors, Contrib. Plasma Phys. 48, 89 (2008).

[12] D. Tskhakaya, R.A. Pitts, W. Fundamenski, T. Eich, S. Kuhn, and JET EFDA Contributors, J. Nucl. Mater. 390-391, 335 (2009).

[13] W. Fundamenski, R. A. Pitts, and JET EFDA contributors, Plasma Phys. Control. Fus. 48, 109 (2006).

[14] C.Z. Cheng and G. Knorr, J. Comput. Phys. 22, 330 (1976).

[15] P. Crispel, P. Degond, and M.-H. Vignal, J. Comput. Phys. 223208 (2007).

[16] P. Degond, F. Deluzet, L. Navoret, An-Bang Sun, and M.-H. Vignal, J. Comput. Phys. 229, 5630 (2010).

[17] R. A. Pitts, P. Andrew, G. Arnoux, T. Eich, W. Fundamenski, A. Huber, C. Silva, D. Tskhakaya, and JET EFDA Contributors, Nucl. Fusion 47, 1437 (2007).

[18] T. Eich, A. Kallenbach, W. Fundamenski, A. Hermann, V. Naulin, JET EFDA contributors, and ASDEX Upgrade team, J. Nucl. Mater. 390-91, 760 (2009).

[19] A. Bergmann, Nucl. Fus. 42, 1162 (2002).

[20] G. Manfredi, S. Mola, and M. R. Feix, Phys. Fluids B 5 (1993).

[21] G. Manfredi, M. Shoucri, M.R. Feix, P. Bertrand, E. Fijalkow, and A. Ghizzo, J. Comput. Phys. 121, 298 (1995).

[22] R. Belaouar, N. Crouseilles, P. Degond, E. Sonnendrücker, J. Sc. Comput. 41, 341 (2009).

[23] E. Fijalkow, Comput. Phys. Commun. 116, 319 (1999).

[24] F. Filbet, E. Sonnendrucker, and P. Bertrand, J. Comput. Phys. 172, 166 (2001). 